\title{
El impacto de la pandemia por coronavirus: percepciones y acciones de los miembros de la Sociedad Mexicana de Oftalmología
}

\section{The coronavirus pandemic impact: perceptions and actions of the members of the Mexican Society of Ophthalmology}

\author{
Juan A. López-Ulloa ${ }^{1,2 *}$, Jaime Soria-Viteri ${ }^{2,3,4}$, Carolina Sardi-Correa ${ }^{2,5}$, \\ Juan P. Olivares-de-Emparan ${ }^{1,2,6}$, Jason Penniecook ${ }^{1,2,7}$, Claudia Palacio-Pastrana ${ }^{8}$, \\ Jesús Jiménez-Román ${ }^{1,6}$ y Van Charles Lansingh ${ }^{1,9,10}$
}

${ }^{1}$ Centro Mexicano de Salud Visual Preventiva, Ciudad de México, México; ${ }^{2}$ The Collaborative Network for Quality in Eye Research (CONQUER); ${ }^{3}$ Universidad de Especialidades Espíritu Santo, Guayaquil, Ecuador; ${ }^{4}$ Universidad Católica Santiago de Guayaquil, Guayaquil, Ecuador; ${ }^{5}$ nstituto Nacional de Investigación en Oftalmología, Medellín, Colombia; ${ }^{6}$ Asociación para Evitar la Ceguera en México, Ciudad de México, México; ${ }^{7}$ Instituto de la Visión, Universidad de Montemorelos, Montemorelos, México; ${ }^{8}$ Sociedad Mexicana de Oftalmología, Ciudad de México, México; ${ }^{9}$ nstituto Mexicano de Oftalmología, Querétaro, México; ${ }^{10} \mathrm{Help} \mathrm{Me} \mathrm{See,} \mathrm{New} \mathrm{York} \mathrm{City,} \mathrm{NY,} \mathrm{USA}$

\section{Resumen}

Objetivo: Analizar el impacto de la emergencia sanitaria por COVID-19 en las percepciones y acciones de los miembros de la Sociedad Mexicana de Oftalmología (SMO) durante los primeros meses. Se describe la asociación entre las características profesionales y la implementación de medidas de bioseguridad publicadas por la SMO. Método: Encuesta en línea, mediante Google ${ }^{\circledR}$ Forms, enviada por correo electrónico a oftalmólogos registrados en la SMO. Se enviaron en total 3296 cuestionarios. Se codificaron dos variables principales para analizar las hipótesis exploratorias: experiencia de los encuestados y tipo de práctica. Resultados: Se obtuvieron 363 respuestas (11.01\%). Al momento de la encuesta, 223 (61.3\%) oftalmólogos continuaban la atención clínica y 140 (38.5\%) habían cesado toda actividad. La principal razón para detener el trabajo clínico fue el temor a convertirse en centros de transmisión. Hubo una asociación entre la mayor experiencia y la interrupción de la atención al paciente ( $p=0.002)$. Continuaron realizando cirugías $107(29.7 \%)$ oftalmólogos. Los participantes con menos de 10 años de experiencia tenían mayor probabilidad de realizar cirugías de emergencia $(p=0.04)$. La medida de seguridad más empleada fue el uso de cubrebocas en los pacientes, seguido del uso de mascarillas N95 por parte de los médicos. Conclusiones: La mayoría de los participantes mantuvieron su trabajo clínico, pero menos de un tercio continuaron las actividades quirúrgicas. Hubo una gran diversidad en las acciones emprendidas y en la adopción de las recomendaciones de la SMO. El uso de equipo de protección personal fue la acción más frecuente entre los participantes.

Palabras clave: Encuesta. Oftalmólogo. Personal de salud. Percepción. COVID-19. SARS-CoV-2.

\section{Abstract}

Purpose: To report the perceptions and actions undertaken by members of the Mexican Society of Ophthalmology (SMO) during the first months of the COVID-19 pandemic. The paper focuses on the association between professional characteristics

Fecha de recepción: 24-07-2020

Fecha de aceptación: 18-11-2020 C.P. 36600, Irapuato, Guanajuato, México E-mail: jlopezulloa @ imoiap.edu.mx DOI: 10.24875/RMO.M2000015
Disponible en internet: $24-12-2020$ Rev Mex Oftalmol. 2021:95(2):46-55 www.rmo.com.mx (http://creativecommons.org/licenses/by-nc-nd/4.0/). 
and the extent of implementation of the recommendations published by the SMO. Methods: An online survey at Google ${ }^{\oplus}$ Forms was sent via email to ophthalmologists registered with the SMO. A total of 3,296 questionnaires were sent. Two principal variables were selected for the purpose of analyzing the exploratory hypotheses: seniority of the respondents and type of practice. Results: A total of 363 replies were obtained (11.01\%). At the time of the survey, 223 ophthalmologists (61.3\%) were continuing to see patients in their clinics, while 140 participants (38.5\%) had ceased all activities. The main reason for stopping clinical work was the fear that the practices would become transmission hubs. There was a statistical association between higher seniority and discontinuing patient care $(p=0.002)$. A total of $107(29.7 \%)$ participants continued performing surgical procedures. Participants with less than 10 years of experience had a higher probability of engaging in emergency surgical procedures $(p=0.04)$. The most commonly employed preventive measure was the use of face masks for patients, followed by the use of $\mathrm{N} 95$ respirators by doctors. Conclusion: The majority of the participants maintained their clinical work; in contrast, less than a third continued surgical activities. There was great diversity in the actions taken and in the adoption of SMO recommendations. The use of personal protective equipment was the most employed security measure.

Key words: Survey. Ophthalmologist. Health care workers. Perception. COVID-19. SARS-CoV-2.

\section{Introducción}

EI SARS-CoV2 (Severe Acute Respiratory Syndrome CoronaVirus 2) se identificó por primera vez, en China, a principios de enero de $2020^{1}$. El 11 de marzo, con casos reportados en más de 110 países, la Organización Mundial de la Salud declaró la enfermedad por coronavirus 19 (COVID-19) como una pandemia ${ }^{2}$. Se han implementado una plétora de medidas de salud pública en todo el mundo para limitar la propagación del virus, entre las que destacan el distanciamiento social, el uso de cubrebocas y mascarillas faciales, y la higiene de manos $^{3}$. Por otro lado, otras medidas han recibido menos énfasis y se han implementado en un grado variable según los países, como lo son la suspensión de actividades comerciales, los toques de queda y la identificación y el rastreo de $\operatorname{casos}^{4,5}$.

En México, la Secretaría de Salud esbozó un enfoque de tres fases para escalar las medidas tomadas de acuerdo con el grado de contagios en los primeros meses de la contingencia. Las fases se definieron según el origen y el número de casos. En la fase 1, los casos se originaron en el extranjero y se importaron al país; la fase 2 correspondía a la transmisión local esporádica; y la fase 3 involucró la detección de brotes importantes en distintos lugares dentro del país 6 . El 24 de marzo se anunció la fase 2 y una semana después se declaró el estado de emergencia de salud pública ${ }^{7}$. El 21 de abril se declaró el inicio de la fase $3^{6}$. A pesar de la preocupante situación en Europa y Asia, el gobierno mexicano no impuso toques de queda como lo hicieron otros países latinoamericanos ${ }^{8-11}$. Como parte de sus esfuerzos para limitar la propagación del virus, el gobierno impuso restricciones a las actividades económicas de la mayoría de las industrias, mientras que al mismo tiempo permitió el libre movimiento y transporte de personas ${ }^{7}$. A la fecha, queda por ver cómo estas acciones impactarán la trayectoria de la pandemia.

En contraste con la situación en otros países, no hubo instrucciones oficiales del gobierno mexicano sobre las acciones recomendadas para médicos y trabajadores de la salud ${ }^{11}$. Numerosas sociedades oftalmológicas internacionales han emitido guías y recomendaciones sobre las medidas que debían tomarse durante esta crisis $^{12-14}$. El Centro Mexicano de Salud Visual Preventiva (CMSVP) ha trabajado en colaboración con la Sociedad Mexicana de Oftalmología (SMO) para brindar sugerencias específicas a los médicos sobre cómo proceder ${ }^{15}$. Las recomendaciones nacionales para oftalmólogos se publicaron por primera vez el 23 de marzo, y se revisan y actualizan continuamente. Sin embargo, la emisión de pautas y recomendaciones no se traduce necesariamente en su implementación. Se han propuesto varias razones para explicar este fenómeno: desacuerdo con las recomendaciones, limitaciones organizativas y falta de conocimiento sobre la disponibilidad de las guías, entre otras ${ }^{16}$.

Como parte de los esfuerzos del CMSVP para brindar asistencia durante esta crisis, se circuló una encuesta para comprender mejor las medidas adoptadas por los oftalmólogos mexicanos durante el estado de emergencia de salud pública declarado por el gobierno. El objetivo del estudio fue reportar las percepciones y las acciones de los miembros activos de la SMO durante los primeros 3 meses de la pandemia de COVID-19. El presente trabajo se centra en el impacto de la COVID-19 en las prácticas oftalmológicas, el grado de implementación de las recomendaciones emitidas por la SMO y la asociación entre dichas medidas y las características profesionales de los participantes. 


\section{Método}

Se desarrolló y distribuyó una encuesta de 21 elementos a través de la plataforma Google ${ }^{\circledR}$ Forms, de manera confidencial. La encuesta se envió por correo electrónico a los oftalmólogos que figuraban en el registro electrónico de la SMO. Se notificó a los encuestados la intención de publicar el trabajo, y el hecho de contestar la encuesta sirvió como consentimiento informado para el estudio. Todos los procedimientos se ajustaron a los principios de la Declaración de Helsinki. El estudio siguió las pautas para informar sobre la investigación basada en encuestas de la Association of American Medical Colleges (AAMC) ${ }^{17}$.

\section{Desarrollo de la encuesta}

Tras revisar la literatura se definieron cinco temas de interés principales: 1) el impacto de la pandemia en las prácticas oftalmológicas; 2) las percepciones de los oftalmólogos sobre los riesgos del coronavirus en sus prácticas; 3) la implementación de medidas preventivas; 4) las actitudes hacia el uso de telemedicina; y 5) las repercusiones económicas de la pandemia en el ámbito oftalmológico. El documento actual informa sobre los tres primeros temas, mientras que los dos últimos se tratarán en una publicación adicional.

Se siguieron los siguientes pasos como mecanismos de validación del instrumento: 1) la encuesta fue revisada y aprobada por tres expertos del CMSVP; 2) se realizó una encuesta piloto antes del lanzamiento de la encuesta en línea; 3) la encuesta fue revisada por miembros del Comité de Ética del Instituto Mexicano de Oftalmología; y 4) la presidente de la SMO, como representante de esta, revisó y aprobó la encuesta. La lista de verificación para el desarrollo de encuestas proporcionada por la AAMC se utilizó en todas estas etapas ${ }^{18}$. Para la elaboración del estudio, los autores basaron algunas preguntas en una encuesta similar realizada por Nair, et al..$^{19}$ en India, que tenía como objetivo medir el efecto de la pandemia de COVID-19 en las prácticas oftalmológicas en ese país.

\section{Variables principales}

El objetivo del estudio fue analizar el impacto de la pandemia en la práctica de los miembros de la SMO, así como su comportamiento en relación con las medidas de bioseguridad y la asociación con sus características profesionales. Se seleccionaron dos variables principales con el fin de analizar las hipótesis exploratorias: antigüedad
Tabla 1. Esquema de la encuesta a oftalmólogos sobre la pandemia de COVID-19

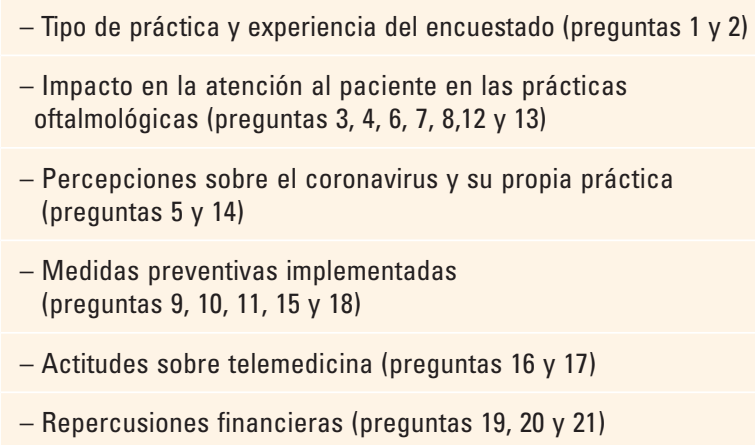

de los encuestados y tipo de práctica. La antigüedad se refiere al número de años ejerciendo la oftalmología después de completar la formación académica formal. El tipo de práctica indica si las principales actividades del encuestado se llevaron a cabo en el sector público o en el privado. La categoría del sector privado se dividió además en aquellos que trabajan en consultorios privados propios o en centros de asistencia privada especializados, una entidad común en México, donde una institución privada sin fines de lucro brinda servicios asequibles a los pacientes. Los participantes que trabajaban en más de un sector se clasificaron como práctica mixta. Se omitieron deliberadamente otras variables referentes a características demográficas, con el fin de centrarse en el entorno profesional de los participantes.

\section{Encuesta final}

El instrumento final constó de 21 ítems: 2 referidos a las variables principales y 19 que recopilaban información relacionada con las cinco áreas de interés definidas anteriormente. El esquema del cuestionario final se muestra en la tabla 1, y en el Anexo se reproduce íntegramente. El cuestionario se distribuyó el 8 de mayo de 2020, 2 semanas después de la declaración de la fase 3. La encuesta permaneció abierta para recibir respuestas durante 3 semanas. Todas las respuestas recibidas se mantuvieron confidenciales.

\section{Tamaño de muestra}

Se invitó a participar a los miembros de la SMO cuyas direcciones de correo electrónico figuraban en su registro oficial. Se enviaron 3296 cuestionarios. Se utilizó un cálculo de muestra para poblaciones finitas y variables categóricas para lograr un margen de error 
Tabla 2. Asociación entre tipo de práctica y experiencia de los encuestados

\begin{tabular}{|l|c|c|c|c|c|}
\hline & $<10$ años $(\mathbf{n}=81)$ & $11-20$ años $(\mathbf{n}=106)$ & $21-30$ años $(\mathbf{n = 8 6 )}$ & $>30$ años $(\mathbf{n = 8 7 )}$ & $\mathbf{p}$ \\
\hline Práctica privada & $21(25.9 \%)$ & $46(43.4 \%)$ & $37(41.6 \%)$ & $61(70.1 \%)$ & $<0.001$ \\
\hline Mixta & $48(59.3 \%)$ & $53(50.0 \%)$ & $40(44.9 \%)$ & $26(29.9 \%)$ \\
\hline Sector público/asistencia privada & $12(14.8 \%)$ & $7(6.6 \%)$ & $12(13.5 \%)$ & $0(0 \%)$
\end{tabular}

del $5 \%$ con un nivel de confianza del 95\%. Se utilizó una calculadora en línea para el cálculo (www.surveysystem.com/sscalc.htm). Se estimó un mínimo de 344 respuestas. El número de respuestas obtenidas por pregunta (n) varió, ya que algunas de ellas solo se incluyeron de acuerdo con las selecciones previas del participante en cada encuesta.

Se pretendía comprender los patrones y las tendencias adoptados por los miembros de la SMO durante la pandemia, así como la influencia de sus características profesionales en la implementación de medidas de bioseguridad. Se determinó que una muestra por conveniencia sería adecuada para este propósito. Esta decisión se basó en una tasa de respuesta del 10\% para las encuestas en línea, una base de datos existente de 3000 oftalmólogos y un cálculo de muestra de 300 participantes.

\section{Análisis estadístico}

Los datos se procesaron utilizando una hoja de cálculo de Microsoft ${ }^{\circledR}$ Excel. La variable «tipo de práctica» se codificó como se describió anteriormente. Para los ítems en los que estaba disponible la selección de respuesta múltiple, se contabilizó cada opción de respuesta y se obtuvo su porcentaje sobre el número total de respuestas. Para la estadística descriptiva se informaron las frecuencias y las proporciones, y se realizó un análisis exploratorio utilizando las pruebas de ji al cuadrado, Fisher y Kruskal Wallis, según fuese el caso. Para realizar el análisis se utilizó el software SPSS ${ }^{\circledR}$ versión 21 (IBM, EE.UU.). La significación estadística se registró con un valor de $p<0.05$ utilizando un análisis de dos colas. Para la creación de la figura se utilizaron R Studio versión 4.01, DataGraph versión 4.6 y Wizard Stats 1.9.41.

\section{Resultados}

La encuesta se envió a 3296 oftalmólogos registrados en la base de correos electrónicos de la SMO. Se obtuvieron 363 respuestas (tasa de respuesta del $11.01 \%$ ) al final de las 3 semanas (1 de junio de 2020).

Con respecto al tipo de práctica, 165 (45.3\%) encuestados trabajaban solo en la práctica privada, 31 (8.5\%) trabajaban en el sector público o en un centro de asistencia privada y $167(45.9 \%)$ trabajaban en ambos. Según la encuesta, $81(22.3 \%)$ tenían de 1 a 10 años de experiencia clínica, 106 (29.2\%) tenían de 10 a 20 años, $89(24.5 \%)$ tenían de 20 a 30 años y 87 (24\%) más de 30 años. Se observó una asociación significativa entre mayor experiencia y trabajo en la práctica privada $(p<0.001)$. En la tabla 2 se muestran el tipo de práctica y la antigüedad de los encuestados.

En el momento del estudio, la mayoría de los participantes $(223,61.3 \%)$ continuaban dando consulta, mientras que 140 (38.5\%) habían cesado sus actividades debido a la pandemia. De estos últimos, 42 (30\%) interrumpieron las visitas cuando se declaró la fase I, $71(50.7 \%)$ durante la fase 2 y $9(6.4 \%)$ al inicio de la fase 3. Cesaron sus prácticas de manera intermitente $18(12.9 \%)$ participantes, independientemente de las declaraciones nacionales. No hubo asociación significativa entre el tipo de práctica y la interrupción de actividades $(p=0.7)$. Por el contrario, en comparación con los más jóvenes, los participantes con mayor experiencia tenían más probabilidades de cesar sus actividades $(p=0.002)$.

Con respecto al impacto de la pandemia en su práctica diaria, de los 223 participantes que continuaron trabajando, en comparación con los números previos a la contingencia, 157 (70.4\%) informaron una disminución del $80 \%$ en el número de pacientes atendidos por mes, $47(21.1 \%)$ informaron una disminución del $50 \%$ y $17(7.6 \%)$ informaron una caída del $20 \%$. Solo $2(0.9 \%)$ no informaron cambios en el número de pacientes.

En cuanto a los motivos para suspender las actividades $(n=129), 54(41.8 \%)$ manifestaron que su principal preocupación era convertirse en un centro de transmisión, 48 (37.2\%) temían contagiarse, 19 (14.7\%) se vieron obligados a cerrar por la caída en la demanda de 
Tabla 3. Asociación entre los motivos de suspensión de las actividades clínicas y la fase de contingencia en México

\begin{tabular}{|l|r|r|r|r|c|}
\hline & Fase I $(\mathbf{n}=38)$ & Fase II $(\mathbf{n}=69)$ & Fase III $(\mathbf{n = 9})$ & Intermitente $(\mathbf{n}=13)$ \\
\hline $\begin{array}{l}\text { Preocupación de convertirse en un centro de } \\
\text { transmisión }\end{array}$ & $24(63.2 \%)$ & $28(40.6 \%)$ & $2(22.2 \%)$ & 0 \\
\hline Miedo al contagio & $9(23.7 \%)$ & $31(44.9 \%)$ & $4(44.4 \%)$ & $4(30.7 \%)$ \\
\hline Caída en la demanda de servicios & $3(7.9 \%)$ & $5(7.2 \%)$ & $2(22.2 \%)$ & $9(69.3 \%)$ \\
\hline Cierre por autoridades locales & $2(5.3 \%)$ & $5(7.2 \%)$ & $1(11.1 \%)$ & 0 \\
\hline
\end{tabular}

servicios, y las autoridades locales pidieron a 8 (6.2\%) que cesaran sus actividades. No respondieron a esta pregunta 11 participantes. Se encontró una diferencia significativa entre las razones para el cese de las actividades clínicas según las fases de la pandemia $(p=0.001)$. En la tabla 3 se resumen estos hallazgos.

De los 223 participantes que continuaron con sus actividades clínicas, la mayoría brindó servicios solo a pacientes urgentes o que requerían seguimiento por riesgo de pérdida de visión (162, 72.6\%). No hubo diferencia estadística entre los tipos de pacientes recibidos y el tipo de práctica, ya fuese pública o privada $(p=0.12)$. Aunque no fue estadísticamente significativo, el análisis mostró una tendencia a que los participantes más jóvenes recibieran todo tipo de pacientes $(p=0.07)$.

Continuaron realizando procedimientos quirúrgicos 107 (29.7\%) participantes. A estos médicos se les preguntó qué tipos de cirugías continuaban practicando. El análisis mostró que los participantes con menos de 10 años de experiencia tenían una mayor probabilidad de participar en procedimientos de emergencia $(p=0.04)$. El análisis también mostró que los que trabajaban en consultorios privados tenían más probabilidades de administrar tratamientos intravítreos dirigidos contra el factor de crecimiento endotelial vascular a todos los casos en comparación con los participantes que trabajaban en el sector público o en centros de asistencia $(p=0.04)$. En la tabla 4 se resumen estos hallazgos.

A la pregunta sobre el riesgo percibido de contagio para los oftalmólogos, $241(66.6 \%)$ respondieron que era mayor, $103(28.5 \%)$ creían que el riesgo era similar y $18(5 \%)$ respondieron que era menor en comparación con otras especialidades médicas. Nuestro análisis no mostró una asociación entre la experiencia y el riesgo percibido de contagio. Asimismo, no hubo asociación entre dicho riesgo percibido y la probabilidad de suspender procedimientos quirúrgicos. Por el contrario, sí la hubo con la suspensión de consultas $(p=0.05)$.
La encuesta preguntó sobre las medidas preventivas actualmente empleadas, así como sobre las que los participantes esperaban adoptar en un futuro. Entre los oftalmólogos que continuaron su práctica (223), la medida más comúnmente empleada fue el uso universal de cubrebocas en todos los pacientes, con 214 (96\%) encuestados declarando haber adoptado esta práctica. La segunda medida más común fue la colocación de mascarillas N95 por parte de los médicos durante sus interacciones con todos los pacientes (149, $66.8 \%$ ). Respecto a las medidas que los participantes adoptarían en el futuro, $332(92.2 \%)$ respondieron que limitarían los acompañantes del paciente, mientras que $321(89.2 \%)$ emplearían pantallas o escudos de lámpara de hendidura. El compromiso de implementar el uso de cubrebocas quirúrgicos por parte de los pacientes en el futuro disminuyó del $96 \%$ al $82.2 \%$. En la figura 1 se resumen estos hallazgos.

\section{Discusión}

La pandemia por SARS-CoV2 ha tenido repercusiones importantes para las sociedades de todo el mundo, y México no es la excepción. En el campo de la salud, ha tenido un efecto significativo en la forma de practicar la oftalmología. El objetivo de esta encuesta fue evaluar las acciones y las actitudes de los miembros de la SMO durante este periodo excepcional. De los resultados obtenidos destaca que, a pesar de que el $72.6 \%$ de los oftalmólogos encuestados continuaron trabajando durante la pandemia, la mayoría reportó una disminución de aproximadamente el $80 \%$ en el flujo de pacientes. Aunque las limitaciones a las actividades económicas en el país no fueron tan restrictivas como los cierres en otras naciones, las limitaciones y el estado de ánimo del público llevaron a una situación sin precedentes y una disminución considerable en la demanda de atención oftalmológica. Es probable que las medidas tomadas durante estos meses continúen implementándose en el futuro previsible. 
Tabla 4. Actividades clínicas y quirúrgicas durante la pandemia COVID-19 por parte de oftalmólogos mexicanos

\begin{tabular}{|c|c|c|c|c|c|}
\hline \multicolumn{6}{|c|}{ Tipo de pacientes atendidos } \\
\hline & \multicolumn{4}{|c|}{ Años de experiencia } & \multirow[t]{2}{*}{ Valor $\mathrm{P}$} \\
\hline & $\begin{array}{c}<10 \text { años } \\
(n=63)\end{array}$ & $\begin{array}{c}\text { 11-20 años } \\
(\mathrm{n}=67)\end{array}$ & $\begin{array}{c}21-30 \text { años } \\
(\mathrm{n}=47)\end{array}$ & $\begin{array}{l}>30 \text { años } \\
(\mathrm{n}=46)\end{array}$ & \\
\hline $\begin{array}{l}\text { Emergencias y seguimiento en pacientes con riesgo de } \\
\text { pérdida de visión }\end{array}$ & $46(73.0 \%)$ & $49(73.1 \%)$ & $35(74.5 \%)$ & $32(69.6 \%)$ & \multirow[t]{3}{*}{0.07} \\
\hline Seguimiento a pacientes subsecuentes & $2(3.2 \%)$ & $2(3.0 \%)$ & $6(12.8 \%)$ & $7(15.2 \%)$ & \\
\hline Todo tipo de pacientes & $15(23.8 \%)$ & $16(23.9 \%)$ & $6(12.8 \%)$ & $7(15.2 \%)$ & \\
\hline \multicolumn{6}{|c|}{ Tip de cirugías practicadas } \\
\hline & $\begin{array}{c}<10 \text { años } \\
(n=30)\end{array}$ & $\begin{array}{l}\text { 11-20 años } \\
(\mathrm{n}=45)\end{array}$ & $\begin{array}{l}21-30 \text { años } \\
(n=20)\end{array}$ & $\begin{array}{l}>30 \text { años } \\
(n=12)\end{array}$ & Valor $P$ \\
\hline Procedimientos de urgencia & $28(93.3 \%)$ & $34(75.6 \%)$ & $14(70.0 \%)$ & $7(58.3 \%)$ & 0.04 \\
\hline Cirugías filtrantes de glaucoma & $6(20.0 \%)$ & $4(8.9 \%)$ & $3(15.0 \%)$ & $1(8.3 \%)$ & 0.54 \\
\hline Cirugías de catarata electivas & $2(6.7 \%)$ & $3(6.7 \%)$ & $4(20.0 \%)$ & $1(8.3 \%)$ & 0.37 \\
\hline Cirugías refractivas & $1(3.3 \%)$ & $1(2.2 \%)$ & $1(5.0 \%)$ & $1(8.3 \%)$ & 0.57 \\
\hline $\begin{array}{l}\text { Cirugías de catarata prioritarias (congénita, traumática, } \\
\text { glaucomas inducidos por cristalino) }\end{array}$ & $3(10.0 \%)$ & $5(11.1 \%)$ & $4(20.0 \%)$ & $3(25.0 \%)$ & 0.43 \\
\hline Párpado y vías lagrimales & $2(6.7 \%)$ & $1(2.2 \%)$ & 0 & 0 & 0.62 \\
\hline Tumores y pacientes oncológicos & 0 & $5(11.1 \%)$ & 0 & 0 & 0.11 \\
\hline Todo tipo de cirugía & 0 & $2(4.4 \%)$ & 0 & $1(8.3 \%)$ & 0.36 \\
\hline \multicolumn{6}{|c|}{ Inyecciones intravítreas de anti-VEGF } \\
\hline & $\begin{array}{l}<10 \text { años } \\
(n=59)\end{array}$ & $\begin{array}{l}11-20 \text { años } \\
\text { (n= 61) }\end{array}$ & $\begin{array}{l}21-30 \text { años } \\
(n=42)\end{array}$ & $\begin{array}{l}>30 \text { años } \\
(n=40)\end{array}$ & Valor $\mathbf{P}$ \\
\hline Cesó la aplicación en todos los casos & $28(48.3 \%)$ & $24(39.3 \%)$ & $23(54.8 \%)$ & $23(57.5 \%)$ & \multirow[t]{3}{*}{0.37} \\
\hline Solo en casos de DMRAE húmeda & $26(43.3 \%)$ & $30(49.2 \%)$ & $14(33.3 \%)$ & $11(27.5 \%)$ & \\
\hline Continuó aplicando tratamiento en todos los casos & $5(8.3 \%)$ & $7(11.5 \%)$ & $5(11.9 \%)$ & $6(15.0 \%)$ & \\
\hline
\end{tabular}

Los encuestados adoptaron una variedad de enfoques durante el brote, y un propósito de este estudio fue asociar dichos enfoques con sus características profesionales. Un número importante había cesado sus actividades clínicas en el momento de la encuesta (38.5\%). No encontramos asociación entre el tipo de práctica y estas acciones $(p=0.7)$, pero sí encontramos que los participantes con mayor antigüedad tenían más probabilidades de interrumpir las actividades en comparación con los más jóvenes ( $p=0.002)$. Para complementar nuestro análisis, queríamos averiguar si la percepción del riesgo podría haber influido en estas decisiones. Logramos encontrar una asociación entre la percepción del riesgo y la suspensión de consultas $(p=0.047)$. Similarmente, Nair, et al. ${ }^{19}$ encontraron que la mayoría de los oftalmólogos en la India perciben un mayor riesgo de contagio al compararse con compañeros de otras especialidades médicas, probablemente por el hecho de llevar a cabo la exploración oftalmológica a menos de $30 \mathrm{~cm}$ de la cara del paciente. Esta percepción de riesgo puede estar infundada, ya que un estudio reciente realizado en Wuhan informó que la incidencia general de COVID19 entre los profesionales de la salud visual fue del $2.52 \%^{20}$, similar a la observada para otras especialidades. Por su parte, sería lógico suponer que los médicos percibirían su propio riesgo de contagio como mayor dependiendo de su edad y que, por lo tanto, los participantes mayores cesarían sus actividades clínicas. Sin embargo, no se observó esta asociación al 




Figura 1. Medidas preventivas tomadas durante la pandemia contrastadas con las que los participantes esperaban adoptar en el futuro cercano. Las barras muestran proporcionalmente el número de respuestas positivas para cada intervención. Las áreas de color gris claro representan a todos los participantes; las áreas de color representan las medidas que se están tomando actualmente y las áreas gris oscuro representan implementaciones planificadas a futuro. Los elementos marcados con un asterisco no figuraban en las recomendaciones publicadas de la Sociedad Mexicana de Oftalmología. Las medidas se agrupan en tres categorías: recomendaciones administrativas (rojo), adaptaciones ambientales (verde) y equipo de protección personal (azul).

analizar las respuestas de la encuesta. Es importante señalar que no hubo preguntas específicas sobre comorbilidad, lo que podría repercutir en la decisión de suspender el trabajo clínico.
Al estudiar los factores que determinaron la suspensión de actividades, se evidenció que los médicos cerraron sus consultorios por diferentes motivos según el momento de su decisión. Se encontró una 
asociación significativa entre estos motivos y las fases de la pandemia $(p=0.001)$. Los participantes estaban más preocupados por que sus prácticas se convirtieran en centros de transmisión durante las etapas iniciales. A medida que la situación empeoraba, la principal razón para suspender las consultas fue el miedo al contagio. Mientras tanto, la mayoría de los que informaron haber interrumpido sus actividades de forma intermitente lo hicieron debido a una caída en la demanda de atención oftalmológica más que por percibir un riesgo para su salud.

En el momento de la encuesta, entre los médicos que mantenían su actividad clínica, el $72.6 \%$ brindaba servicios solo a pacientes con emergencias y seguimiento a aquellos con riesgo de pérdida de visión. Incluso cuando la demanda de servicios flaqueó, los participantes de la encuesta se mostraron reacios a recibir pacientes cuyas necesidades no eran críticas. Esto refleja los hallazgos del estudio de Nair, et al. ${ }^{19}$, en el que hasta el $82 \%$ de los doctores encuestados en India continuaba atendiendo pacientes urgentes a pesar del cese de actividades. Encontramos una asociación en nuestros datos con la experiencia de los médicos, ya que los participantes más jóvenes, en particular aquellos con menos de 20 años de experiencia, tenían una mayor probabilidad de recibir pacientes con cualquier tipo de problema.

En comparación con las consultas, las actividades quirúrgicas se vieron aún más afectadas: el $70 \%$ de los participantes de nuestra encuesta suspendieron cualquier tipo de procedimiento quirúrgico. Los que continuaron operando disminuyeron considerablemente su actividad. Los cirujanos activos operaban predominantemente casos de urgencia. Se encontraron conductas similares en Israel, donde Wasser, et al. ${ }^{21}$ mostraron que la mayoría de los oftalmólogos $(72.7 \%)$ creían que la actividad quirúrgica debería limitarse a las urgencias. Observamos una clara relación entre la edad de los cirujanos y su renuencia a realizar cirugías, ya que los participantes con menos de 10 años de experiencia tenían una mayor probabilidad de realizar todo tipo de cirugía $(p<0.001)$. Esto puede ser indicativo de que los cirujanos se abstienen de cirugías electivas según lo indicado por las guías oficiales publicadas por la SMO.

En cuanto a las medidas preventivas de bioseguridad, encontramos que las dos más comúnmente empleadas fueron el uso de cubrebocas en todos los pacientes y de mascarillas N95 por parte de los médicos. Las medidas que se consideraron con mayor probabilidad de implementarse en un futuro fueron las de limitar el número de acompañantes durante las visitas clínicas y adquirir pantallas o escudos para las lámparas de hendidura. Curiosamente, el enfoque de las medidas pasó del uso de equipo de protección personal (EPP) a las adaptaciones ambientales. Este cambio podría reflejar que algunas medidas se consideran de bajo esfuerzo y alto valor. Las adaptaciones ambientales pueden requerir una mayor inversión inicial y, por lo tanto, es posible que no se adopten tan fácilmente durante las primeras etapas de la pandemia. Cabe destacar que el porcentaje de participantes que requerirían que todos los pacientes usaran cubrebocas en el futuro disminuyó en un 14\% en comparación con las medidas actualmente empleadas. Es posible que este cambio se relacione con las adaptaciones ambientales previstas.

La figura 1 resume las medidas adoptadas por los integrantes de la SMO que participaron en la encuesta. Las medidas preventivas se agrupan en tres categorías: EPP, adaptaciones ambientales y recomendaciones administrativas. Esta clasificación sigue las pautas internacionales establecidas por diversos países asiáticos, como se discutió en un artículo anterior publicado por el Grupo CONQUER ${ }^{22}$. La categorización de las medidas en dominios discretos permite un enfoque sistemático de las adaptaciones de seguridad necesarias durante esta crisis. Hong Kong y China aplicaron este enfoque durante la actual pandemia, así como durante el brote de SARS-CoV en $2002^{23}$.

Al momento de la encuesta, la SMO ya había publicado guías oficiales para la práctica oftalmológica durante la pandemia. Aunque dichas guías presentaban recomendaciones sobre EPP y adaptación de instalaciones, se observó que estas no fueron seguidas por todos los participantes de la encuesta, a pesar de su membresía a la SMO. Aunque ilustrativos, los resultados de nuestra encuesta no pueden tomarse como representativos del alcance de la implementación de estas pautas, ya que no hubo una pregunta específica sobre el conocimiento de las guías.

Utilizando el registro de la SMO se logró una tasa de respuesta del $11 \%$. Si bien se obtuvo una muestra representativa, entendemos que los resultados no pueden extrapolarse a toda la población de oftalmólogos del país. Aunque no se pueden obtener conclusiones sobre esta muestra, sí se pueden apreciar patrones y tendencias. Tales tendencias podrían permitir una comprensión más profunda de la justificación para la implementación de determinadas medidas de bioseguridad. Este estudio buscó patrones de comportamiento entre los miembros de la SMO y su asociación con 
características profesionales específicas. Se logró encontrar una serie de asociaciones estadísticamente significativas entre tales características y la implementación de ciertas medidas. Una tasa de respuesta del $10 \%$ para encuestas en línea dentro de un universo definido es adecuada para percibir temas generales. La literatura internacional reconoce la validez de los estudios con una tasa de respuesta inferior al $20 \%$, en particular con encuestas en línea ${ }^{24,25}$. Una encuesta reciente realizada por la American Academy of Ophthalmology reportó una tasa de respuesta del $18 \%$, a pesar de sus considerables recursos y poder organizativo.

Otra limitación de nuestro estudio fue la falta de información sobre la capacidad de toma de decisiones de los encuestados. En una situación tan dinámica, el tiempo de respuesta de la encuesta fue necesariamente limitado. Además, las conductas reportadas en la encuesta fue la de los propios participantes y dependen en parte de la honestidad y la memoria de estos, por lo que puede haber sesgos.

A pesar de estas limitaciones, los resultados obtenidos brindan información valiosa sobre las percepciones y las acciones de los miembros de la SMO durante el periodo inicial de la pandemia de COVID-19. Considerando el carácter extraordinario de esta época y la importancia de tener datos nacionales para orientar las futuras guías, consideramos que este estudio es válido para generar hipótesis sobre las conductas y la implementación de medidas de seguridad por parte de los oftalmólogos mexicanos en tiempos de pandemia.

Si bien no fue un estudio transversal completo, este tipo de encuestas pueden aportar datos útiles para futuras investigaciones y para la creación de políticas y directrices nacionales. Es posible utilizar instrumentos similares de manera periódica para incrementar el rendimiento de datos y apoyar la formulación de una estrategia nacional para la práctica de la oftalmología. Reconocemos y recomendamos la realización de estudios para conocer más sobre las conductas adoptadas en nuestro medio en tiempos de contingencia sanitaria. La pandemia aún no está menguando y todo parece apuntar a una situación prolongada que requerirá cambios significativos en nuestra práctica.

\section{Conclusión}

A pesar de una disminución significativa en el flujo de pacientes y de los temores asociados a la pandemia de COVID-19, la mayoría de los participantes de la encuesta no cesaron su actividad clínica y continuaron atendiendo pacientes, en especial aquellos en situaciones de urgencia. Las medidas preventivas enumeradas en las guías de la SMO no se habían adoptado en su totalidad; sin embargo, se espera que la implementación de las recomendaciones aumente significativamente en el futuro. La experiencia y el tipo de práctica podrían estar asociados con las acciones tomadas durante este periodo extraordinario. Recomendamos dar prioridad a la implementación de estas medidas de bioseguridad con independencia de la percepción individual de riesgo.

\section{Conflicto de intereses}

Los autores declaran no tener ningún conflicto de intereses.

\section{Responsabilidades éticas}

Protección de personas y animales. Los autores declaran que para esta investigación no se han realizado experimentos en seres humanos ni en animales.

Confidencialidad de los datos. Los autores declaran que en este artículo no aparecen datos de pacientes.

Derecho a la privacidad y consentimiento informado. Los autores declaran que en este artículo no aparecen datos de pacientes.

\section{Bibliografía}

1. Khanna RC, Honavar SG. All eyes on coronavirus - what do we need to know as ophthalmologists. Indian J Ophthalmol. 2020;68:549-53.

2. World Health Organization. Coronavirus disease 2019 - Situation report 51. WHO Website, Coronavirus Situation Reports. (Consultado el 23 de mayo de 2020.) Disponible en: https://www.who.int/docs/default-source/ coronaviruse/situation-reports/20200311-sitrep-51-covid-19.pdf?sfvrsn=1ba62e57_10.

3. Chu DK, Âkl EA, Duda $\mathrm{S}$, Solo K, Yaacoub $\mathrm{S}$, Schünemann $\mathrm{HJ}$; COVID-19 Systematic Urgent Review Group Effort (SURGE) study authors. Physical distancing, face masks, and eye protection to prevent person-to-person transmission of SARS-CoV-2 and COVID-19: a systematic review and meta-analysis. Lancet. 2020; 395:1973-87.

4. Our World in Data. Statistics and research: coronavirus (COVID-19) testing. Our World in Data, University of Oxford. (Consultado el 15 de junio de 2020.) Disponible en: https://ourworldindata.org/coronavirus-testing.

5. Gobierno de México. Comunicado Técnico Diario. Información internacional y nacional sobre nuevo coronavirus con corte al 23 de mayo de 2020. (Consultado el 20 de abril de 2020.) Disponible en: https://coronavirus.gob.mx/2020/05/23/conferencia-23-de-mayo/.

6. Gobierno de México. Todo sobre el COVID-19. (Consultado el 3 de junio de 2020.) Disponible en: https://coronavirus.gob.mx.

7. Secretaría de Gobernación México. Acuerdo por el que se declara como emergencia sanitaria por causa de fuerza mayor, a la epidemia de enfermedad generada por el virus SARS-CoV2 (COVID-19). Diario Oficial de la Federación. (Consultado el 12 de mayo de 2020.) Disponible en: https://www.gob.mx/sre/documentos/80689.

8. Ministerio de Salud Argentina. Nuevo coronavirus COVID-19. (Consultado el 20 de junio de 2020.) Disponible en: https://www.argentina.gob.ar/salud/coronavirus-COVID-19.

9. Gobierno de Chile. Plan de acción coronavirus COVID-19. (Consultado el 12 de mayo de 2020.) Disponible en: https://www.gob.cl/coronavirus/cifrasoficiales/ 
10. Ministerio de Salud Pública del Ecuador. Coronavirus Ecuador. (Consultado el 12 de mayo de 2020.) Disponible en: https://coronavirusecuador.com/

11. Ministerio de Salud y Protección Social de Colombia. Nuevo coronavirus COVID-19. (Consultado el 10 de mayo de 2020.) Disponible en: https:// www.minsalud.gov.co/salud/publica/PET/Paginas/Covid-19_copia.aspx.

12. Sociedad Española de Oftalmología. Recomendaciones para la atención a pacientes oftalmológicos en relación con emergencia COVID-19. (Consultado el 14 de mayo de 2020.) Disponible en: https://www.oftalmoseo. com/documentacion/comunicado_conjunto_oftalmologia_covid19.pdf.

13. American Academy of Ophthalmology. New recommendations for urgent and nonurgent patient care. (Consultado el 29 de abril de 2020.) Disponible en: https://www.aao.org/headline/new-recommendations-urgent-nonurgent-patient-care.

14. American Academy of Ophthalmology. Alert: Important coronavirus updates for ophthalmologists. (Consultado el 2 de mayo de 2020.) Disponible en: https://www.aao.org/headline/alert-important-coronavirus-context.

15. Olivares de Emparan JP, Garza-León M, García-Aguirre G, Azcárate-Coral T, Penniecook JA, Lansingh VC, et al. Recomendaciones para el manejo de pacientes que requieren atención oftalmológica durante la pandemia de SARS-CoV-2. Rev Mex Oftalmol. 2020;94:103-12.

16. Lugtenberg M, Zegers-Van Schaick JM, Westert GP, Burgers JS. Why don't physicians adhere to guideline recommendations in practice? An analysis of barriers among Dutch general practitioners. Implement Sci. 2009;4:54.

17. Artino AR, Durning SJ, Sklar DP. Guidelines for reporting survey-based research submitted to academic medicine. Acad Med. 2018;93:337-40.
18. Gehlbach $H$, Artino AR. The survey checklist (Manifesto). Acad Med 2018;93:360-6.

19. Nair AG, Gandhi RA, Natarajan S. Effect of COVID-19 related lockdown on ophthalmic practice and patient care in India: results of a survey. Indian J Ophthalmol. 2020;68:725-30.

20. Qiao C, Zhang H, He M, Ying G, Chen C, Song Y, et al. Symptomatic COVID-19 in eye professionals in Wuhan, China. Ophthalmology. 2020;127:1268-70.

21. Wasser LM, Assayag E, Tsessler M, Weill Y, Becker-Cohen M, Zadok D. Response of ophthalmologists in Israel to the novel coronavirus (2019nCoV) outbreak. Graefes Arch Clin Exp Ophthalmol. 2020:258:1419-26.

22. Emparan JPO, Sardi-Correa C, López-Ulloa JA, Viteri-Soria J, Penniecook JA, Jimenez-Román J, et al. COVID-19 and the eye: how much do we really know? A best evidence review. Arq Bras Oftalmol. 2020;83:250-61.

23. Lai THT, Tang EWH, Chau SKY, Fung KSC, Li KKW. Stepping up infection control measures in ophthalmology during the novel coronavirus outbreak: an experience from Hong Kong. Graefes Arch Clin Exp Ophthalmol. 2020;258:1049-55.

24. Morton SM, Bandara DK, Robinson EM, Carr PEA. In the $21^{\text {st }}$ Century, what is an acceptable response rate? Aust $\mathrm{N} \mathrm{Z} \mathrm{J} \mathrm{Public} \mathrm{Health.}$ 2012;36:106-8.

25. American Academy of Ophthalmology. Optometry in the American Academy of Ophthalmology. Communication by Board of Trustees. (2020, September 28). (Consultado el 11 de octubre de 2020.) Disponible en: https://www.aao.org/about/policies/optometry-academy-2020. 